Ergod. Th. \& Dynam. Sys. (1988), 8, 531-553

Printed in Great Britain

\title{
Geodesic flow on the two-sphere, Part I: Positive measure entropy
}

\author{
VICTOR J. DONNAY \\ Department of Mathematics, Princeton University, Princeton, NJ 08544, USA
}

(Received 20 June 1987 and revised 28 July 1988)

\begin{abstract}
A $C^{\infty}$ metric is constructed on $S^{2}$ whose geodesic flow has positive measure entropy.

By the uniformization theorem, we can induce a metric of constant negative curvature on the sphere minus three points. Each deleted point gives rise to a cusp going to infinity, which we cut off at some finite point and replace in a smooth way with a cap formed from a surface of revolution. The cap that we use has the property that a diverging family of geodesics that enters the cap will focus once while in the cap and then again be diverging when leaving the cap. The Clairaut integral of motion on a surface of revolution helps us to design this cap.

Using Wojtkowski's invariant cone-field technique, we show that almost every point has a non-zero Lyapunov exponent. Positive entropy then follows by Pesin's formula. Our construction can be applied to surfaces of any genus yielding similar results.
\end{abstract}

\section{Introduction}

On a compact surface $M$ of negative curvature, the geodesic flow behaves stochastically. The original results in this field go back to Hedlund and Hopf, who showed, respectively, that the flow has dense orbits and is ergodic. A geodesic is determined by a point and direction, so the geodesic flow $g_{t}$ occurs in the unit tangent bundle $S M$, and a dense orbit comes arbitrarily close to every point and direction. Ergodicity is meant relative to the invariant Liouville measure $\mu$. These results are only applicable to surfaces of genus $g \geq 2$ since, by the Gauss-Bonnet theorem, the average curvature over the surface equals $2 \pi(2-2 g)$.

Do there exist metrics on the sphere, $g=0$, and torus, $g=1$, for which the geodesic flow behaves stochastically? On the standard sphere, the geodesics are given by the great circles and are all periodic. On the flat torus, $K \equiv 0$, the direction of a geodesic stays constant, the flow is an integrable system and $S M$ decomposes into invariant tori. Under small perturbations of the flat metric, the K.A.M. theory asserts that some of the invariant tori continue to exist, so the system remains non-ergodic.

In this paper, we show that the sphere and torus can be given smooth metrics for which the geodesic flow has positive measure entropy. Positive entropy does not imply ergodicity. Rather, by results of Pesin [16], it implies that the system has components of positive measure on which the flow is ergodic and on which it exhibits the very strongest stochastic behaviour. 
THEOREM 1. Every compact, orientable surface can be given a $C^{\infty}$ Riemannian metric for which the geodesic flow has positive measure entropy.

For surfaces of genus $g \geq 2$ this result is well known since the geodesic flow on a surface of negative curvature is Anosov [1].

For our examples, the geodesic flow is actually ergodic [9]. Applying a perturbation argument to our examples, K. Burns and M. Gerber [6] have produced analytic metrics on the sphere and torus whose geodesic flow has positive entropy and is ergodic. Their proof of ergodicity, which is different from ours, is more general, applying to both the $C^{\infty}$ and analytic examples.

The sphere we construct is an abstract surface; it can not be isometrically embedded in $\mathbb{R}^{3}$. Do there exist spheres embeddable in $\mathbb{R}^{3}$, or better yet, convex spheres in $\mathbb{R}^{3}$ whose geodesic flow is ergodic? At present, the answer is not known.

To construct our example, we start by deleting three (one) or more points from the sphere (torus). By the uniformization theorem, the universal cover of this punctured surface is the disk. The Poincare metric on the disk induces a metric of constant negative curvature on the punctured surface. A theorem of Hilbert [7] asserts that a complete surface of constant negative curvature cannot be embedded in $\mathbb{R}^{3}$. However, in a neighborhood of a deleted point, we can embed the surface, getting a rotationally symmetric cusp (pseudo-sphere) which goes to infinity. We cut off the end of the cusp along a circle and replace it with a cap (figure 1). In the transitional region, where the $K \equiv-1$ surface has been symmetrically tapered to attach to the cap, the curvatue should satisfy $K<0$.

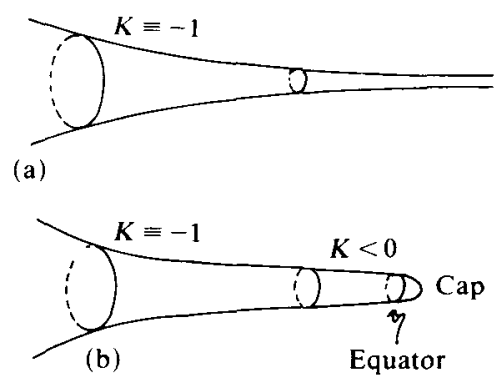

Figure 1. (a) Cusp. (b) Cusp with cap.

In this approach we follow $\mathrm{R}$. Osserman who, using a half-sphere for the cap, created a $C^{1}$, but not $C^{2}$, metric on the sphere whose geodesic flow was ergodic with positive entropy [15]. His proof relied heavily on the symmetry of a half-sphere [see Appendix A.3]. We use a surface of revolution for the cap and are thereby able to maintain smoothness.

For geodesic flow, entropy is a measure of the average exponential rate at which nearby geodesics diverge from one another. A family of geodesics, a variation, that is initially diverging in the negatively curved region will continue to diverge until it reaches a cap. The positive curvature in the cap causes the geodesics to converge. We want a cap that makes the geodesics focus before leaving the cap, so that the variation will again be diverging when it returns to the negatively curved region (figure 2). The variation will then continue to diverge until its next return to the cap. 


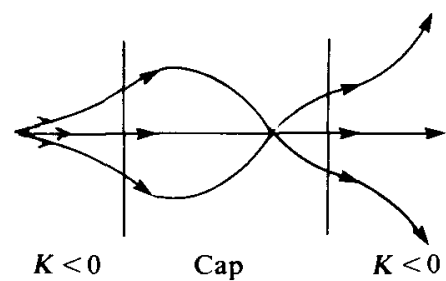

FIGURE 2. Diverging variation focuses in cap.

On a surface of revolution, the geodesic flow has an integral of motion, the so-called Clairaut Integral. This integral allows us to determine explicitly the motion of geodesics in the cap and thereby design a cap that focuses variations in the desired way (\$\$3-6). We call such a cap a focusing cap.

Using M. Wojtkowski's method of invariant cones $[17,18]$, we show that the geodesic flow on our surface has almost everywhere non-zero Lyapunov exponents $(\S 8)$. By Pesin's formula [16] $(\S 2)$, this implies that the measure entropy is positive.

One can also apply this construction of deleting points, but now one or more, and adding caps to a surface of genus $g \geq 2$. The resulting metric will have conjugate points ( $\$ 2$ and Remark 6.2 ), and the geodesic flow will have non-zero Lyapunov exponent almost everywhere. The previous examples of stochastic geodesic flow on surfaces of genus $g \geq 2$ came from metrics without conjugate points [2].

Using our focusing cap, we give a second example of a metric on the torus: a flat torus with cap (figure 3 ). Here the curvature outside the cap is predominately $K \equiv 0$, rather than $K \equiv-1$ as in the first example. This example illustrates the more general conditions under which a surface with focusing caps will have non-zero Lyapunov exponent almost everywhere (Theorem 8.5).

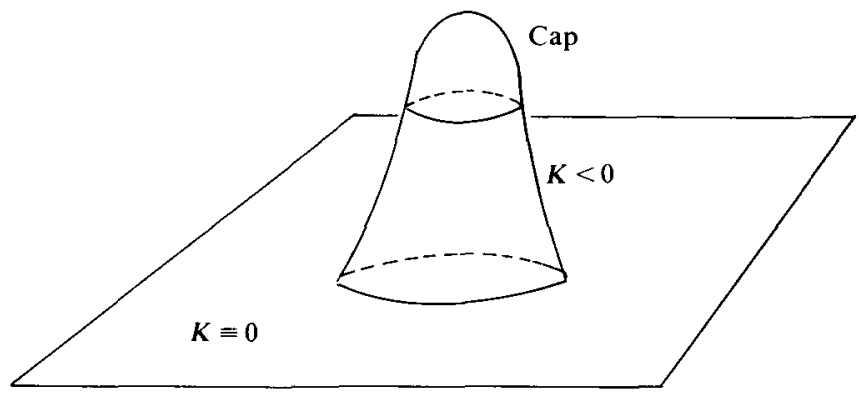

Figure 3. Flat torus with cap.

If we weaken the conditions on our cap, we can still produce positive entropy providing we are more careful in how we attach the cap (\$9).

The flat torus with cap is similar to an example constructed by Bangert [4]. He was studying the question of when the invariant tori of an integrable Hamiltonian system break up. In relating this problem to our example $(\$ 10)$, we discuss the effect non-focusing caps have on the geodesic flow.

Finally, we construct a light-bulb shaped cap $(\$ 11)$ which gives a flow that has positive entropy but is non-ergodic. This example illustrates that integrable and stochastic motion can co-exist within one system. 
Our focusing argument was motivated by Wojtkowski's work on billiards [18] and thus provides further evidence of the link between billiards and geodesic flow on surfaces. One thinks of a billiard table as a two-sided surface with 'infinite' curvature along the boundary. A billiard trajectory on the table gives rise to a geodesic on this 'surface'. A convex boundary component corresponds to positive curvature; reflection at the boundary causes a diverging family of trajectories to converge. Hence, we think of a convex table as analogous to a (convex) sphere. Bunimovitch [5] and Wojtkowski have constructed billiard tables with convex boundary components whose flows behave stochastically. In their examples, the boundary is never smoother than $C^{1}$. Indeed, for a smooth, strictly convex boundary, Lazutkin [12] has shown that the flow cannot be ergodic, although it might have positive entropy. Thus it appears that stochastic behaviour is more easily produced on a sphere than on a convex billiard table.

Acknowledgements. Many of the results in this paper are contained in the author's $\mathrm{PhD}$ thesis [8]. The thesis was supervised by Professor Peter Sarnak who suggested the topic and offered help and advice while the work was in progress. The author also benefited from discussions with Professors Y. Katznelson, J. Moser and D. Ornstein, as well as with P. Doyle and L. Flaminio. The referee's comments were helpful in revising the paper.

The author thanks the Mathematics Department, Stanford University and the Forschungsinstitut für Mathematik, E.T.H. Zürich, where he was a visitor while doing this work.

\section{Background Material}

\subsection{Lyapunov exponents}

Given the Riemannian metric $\langle$, ) on $M$, one canonically induces a metric on $S M$, also denoted by \langle\rangle$[10]$. Let $\mathscr{K}: T(S M) \rightarrow S M$ be the connection map and $\pi: S M \rightarrow M$ the projection map. For $\xi, \eta \in T_{x}(S M)$,

$$
\langle\xi, \eta\rangle=\langle d \pi \xi, d \pi \eta\rangle_{\pi x}+\langle\mathscr{K} \xi, \mathscr{K} \eta\rangle_{\pi x} .
$$

We describe this metric more fully when discussing Jacobi fields. This metric induces a norm \|\| on $T(S M)$.

Define the Lyapunov exponent $\lambda(x, \xi)$ at the point $x$ in the direction $\xi$ by

$$
\lambda(x, \xi)=\varlimsup_{t \rightarrow \infty} \frac{1}{t} \ln \left\|D g_{t} \xi\right\| .
$$

By linearity, $\lambda$ can assume at most 3 distinct values $\lambda^{-}(x) \leq \lambda^{0}(x) \leq \lambda^{+}(x)$ on $T_{x}(S M)$. The Multiplicative Ergodic Theorem [14], applied to our system, implies that for almost every $x \in S M$, the lim sup in (2) is actually a limit for all $\xi \in T_{x}(S M)$ and that $\lambda^{-}(x)=-\lambda^{+}(x)$.

Pesin's formula [16] gives the measure entropy $h_{\mu}$ as the average of the Lyapunov exponents,

$$
h_{\mu}=\int_{S M} \lambda^{+}(x) d \mu(x),
$$


so that positive entropy follows if, on a set of positive measure in $S M$, the Lyapunov exponent $\lambda^{+}$is positive.

\subsection{Variations}

Using the metric (1), we define an orthonormal, right-handed frame $\left\{V, V^{\perp}, \Phi\right\}$ for $T_{x}(S M)$. The vector $V$ is in the flow direction,

$$
V=\left.\frac{d}{d t}\right|_{t=0} g_{t} x .
$$

The horizontal subspace, defined to be the kernel of the operator $\mathscr{K}$, is spanned by $\left\{V, V^{\perp}\right\}$ and is isomorphic to $T_{\pi x} M$. The vertical subspace is the kernel of $d \pi$ and is spanned by $\{\Phi\}$.

To understand this frame geometrically, think of a tangent vector $\xi \in T_{x}(S M)$ as a family of geodesics, a variation. Let $\gamma(s)=(\pi \gamma(s), v(s)), s \in[-\varepsilon, \varepsilon]$, be a curve in $S M$ going through $x$ with tangent vector $\xi, \gamma(0)=x$ and $\gamma^{\prime}(0)=\xi$. Viewed on the surface $M, \gamma(s)$ consists of a curve of basepoints $\pi \gamma(s)$ with vectors $v(s)$ attached at every point. We call $(\pi \gamma(s), v(s))$ a variation based at $x$ and identify it with the vector $\xi \in T_{x}(S M)$. (To be precise, we should identify $\xi$ with the germ of the variation.)

The component of $\xi$ in the horizontal subspace can be identified with the tangent to the curve of basepoints,

$$
d \pi \xi=\left.\frac{d}{d s}\right|_{s=0} \pi \gamma(s) .
$$

The vertical component is given by the covariant derivative of the vector field $v(s)$ in the direction of the curve $\pi \gamma(s)$,

$$
\mathscr{K} \xi=\left.D_{\pi \gamma(s)} v(s)\right|_{s=0} .
$$

We think of $\xi$ as having basepoint (horizontal) and angle (vertical) components.

\subsection{Jacobi fields}

The vectors in the perpendicular subspace of $T_{x}(S M)$, the span of $\left\{V^{\perp}, \Phi\right\}$, are identified with special variations: the (perpendicular) Jacobi fields based at $x$. Let $\gamma_{x}$ be the geodesic on $M$ determined by $x$ and $n(t)$ a continuous field of unit normals along $\gamma_{x}$. Let $J(t)$ be a solution of the Jacobi equation

$$
J^{\prime \prime}(t)+K(t) J(t)=0,
$$

where $K(t)$ is the curvature along the geodesic $\gamma_{x}, K(t)=K\left(\gamma_{x}(t)\right)$. Then $J(t) n(t)$ is a Jacobi field along $\gamma_{x}$, i.e. a vector field obtained from a variation of geodesics through $\gamma_{x}$ [7]. A solution to (4) is uniquely determined by its initial conditions $\left(J(0), J^{\prime}(0)\right)$. We refer to the pair of initial conditions as the Jacobi field. We say the Jacobi field is diverging if $J^{\prime} / J \geq 0$.

To $\xi$ in the perpendicular subspace of $T_{x}(S M)$,

$$
\xi=\xi_{1} V^{\perp}+\xi_{2} \Phi
$$

we associate the Jacobi field

$$
J(0)=\xi_{1}, \quad J^{\prime}(0)=\xi_{2} .
$$


The variation $\gamma(s)=(\pi \gamma(s), v(s))$ corresponding to $\xi$ determines a 1-parameter family of geodesics $g_{1} \gamma(s)$ and hence a Jacobi field,

$$
J(t) n(t)=\left.\frac{d}{d s}\right|_{s=0} \pi \circ g_{t} \gamma(s) .
$$

The value $J(0)$ is given by the basepoint component $\xi_{1}$ of $\xi$. By interchanging order of differentiation, one finds that

$$
\begin{aligned}
J^{\prime}(0) & =\left.\frac{D}{D t}\right|_{t=0} J(t) n(t) \\
& =\left.D_{\pi \gamma(s)} v(s)\right|_{s=0} \\
& =\text { angle component } \xi_{2} \text { of } \xi
\end{aligned}
$$

The vector $D g_{1} \xi$ is in the perpendicular subspace of $T_{g_{t} x}(S M)$ and has norm

$$
\left\|D g_{t} \xi\right\|^{2}=J^{2}(t)+J^{\prime 2}(t)
$$

(for details, see Eberlein [10]).

Definition 2.1. Two points $x$ and $y=g_{t_{*}} x$ are conjugate (focal) along the geodesic $\gamma_{x}$ if there is a solution to (4) with $J(0)=0, J^{\prime}(0)=1$ and $J\left(t_{*}\right)=0\left(J^{\prime}\left(t_{*}\right)=0\right)$. A surface has no conjugate (focal) points if no two points are conjugate (focal) along any geodesic. Non-positive curvature implies no focal points which implies no conjugate points.

\subsection{Riccati equation}

If $\left(J(t ; x), J^{\prime}(t ; x)\right)$ is a Jacobi field based on $x$, we call the function

$$
u(t ; x)=\frac{J^{\prime}}{J}(t ; x)
$$

a Riccati solution. At those times when $J \neq 0, u$ satisfies the Riccati equation

$$
u^{\prime}(t ; x)=-K(t ; x)-u^{2}(t ; x),
$$

where $K(t ; x)=K\left(\pi \circ g_{x} x\right)$. When $J(t)=0$, i.e. $J$ focuses, we identify plus and minus infinity and set $u(t)= \pm \infty$. For $K \leq 0$, the behaviour of Riccati solutions is well understood [13].

\section{Surface of revolution}

In this section, we describe the basic properties of the geodesic flow on a surface of revolution. These properties show that for certain caps the geodesic flow on the complete surface is clearly non-ergodic. So as not to role out the possibility of an ergodic system, we require that the caps satisfy a simple condition.

We define a surface of revolution in terms of geodesic polar coordinates $(l, \theta)$, with $l \in[0, L], \theta \in[0,2 \pi)$. In these coordinates, the surface is determined by the function $r(l)$, which we require to be $C^{\infty}$, that gives the radius of the cap as a function of distance $l$ from the top of the cap. The metric is then

$$
d s^{2}=d l^{2}+r^{2}(l) d \theta^{2} .
$$

At the top of the cap, $r(0) \stackrel{=}{=} 0$ and $r^{\prime}(0)=1$. The curvature $K$ is given by $K(l)=$ $-r^{\prime \prime}(l) / r(l)$. We call the base of the cap, the parallel $r(L)=\mathrm{R}$, the equator. 
In these coordinates, the equations of motion for a geodesic $(l(t), \theta(t))$ are

$$
\begin{aligned}
& \theta^{\prime \prime}(t)+2 \frac{r^{\prime}(l(t))}{r(l(t))} \theta^{\prime}(t) l^{\prime}(t)=0, \\
& l^{\prime \prime}(t)-r(l(t)) r^{\prime}(l(t)) \theta^{\prime 2}(t)=0 .
\end{aligned}
$$

The meridians $\theta=$ constant are geodesics. A necessary and sufficient condition for a parallel $r(l)=$ constant to be a geodesic is that $(d r / d l)(l)=0$.

The geodesic flow on a surface of revolution has an integral of motion, the Clairaut Integral [7]. Let $\phi$ denote the angle a tangent vector makes with the parallel $r=$ constant. Then

$$
r \cos \phi=\text { constant along orbits. }
$$

The integral follows by noting that $\cos \phi=r \theta^{\prime}$ and that, by (10), $r^{2} \theta^{\prime}$ is constant along orbits.

Using the Clairaut Integral, we can describe qualitatively the behaviour of geodesics in the cap. Suppose a geodesic $\gamma_{\phi_{0}}$ starts on the equator with angle $\phi_{0} \in(0, \pi)$. Its behaviour is independent of the coordinate $\theta$. The value $c$ of the Clairaut Integral is given by

$$
c\left(\phi_{0}\right)=R \cos \phi_{0}=r(L) \cos \phi_{0} .
$$

As the geodesic moves through the cap, (11) implies that $\phi$ decreases (increases) as $r$ decreases (increases). The geodesic will climb the cap until it first approaches a point $l_{\min }$ for which $r\left(l_{\min }\right)=c\left(\phi_{0}\right)$. For $l>l_{\min }$, the angles $\phi\left(\gamma_{\phi_{0}}(l)\right)$ are positive.

If $\gamma_{\phi_{0}}$ reaches $r\left(l_{\min }\right)$, then by (11) one has $\phi\left(l_{\min }\right)=0$; the geodesic has flattened out. Solutions of the geodesic equations are unique, so the parallel $r\left(l_{\min }\right)$ cannot be a geodesic. By symmetry, the geodesic $\gamma_{\phi_{0}}$ will then descend the cap, crossing the equator with angle $-\phi_{0}$.

If $\gamma_{\phi_{0}}$ does not reach the parallel $r=r\left(l_{\text {min }}\right)$, then $\gamma_{\phi_{0}}$ becomes asymptotic to it. Uniqueness of solutions implies that this parallel is a geodesic, and so

$$
\frac{d}{d l} r\left(l_{\min }\right)=0 \text {. }
$$

Now consider a geodesic $\gamma_{\phi_{0}, r_{0}}$ that starts inside the cap on a parallel $r_{0}=r\left(l_{0}\right)$, $l_{0}<L$, with angle $\phi_{0}$. If its Clairaut Integral, $c\left(r_{0}, \phi_{0}\right)=r_{0} \cos \phi_{0}$, is greater than $R$, the radius of the equator, then the preceding analysis implies that the geodesic will turn around before reaching the equator and thus spend all its time in the cap. Such a situation would occur if $r(l)$ had a local maximum at $l_{0}$ (see the light-bulb example in \$ 11 ).

If the geodesic flow on the complete surface is to be ergodic, then geodesics must not get stuck in the cap.

Proposition 3.1. If $r(l)$ satisfies

$$
\frac{d}{d l} r(l)>0 \text { for } l \in[0, L),
$$

then every geodesic that enters or starts inside the cap will leave the cap. 


\section{Rotation function $\Delta \theta(\phi)$}

For a cap satisfying (13), any geodesic that enters the cap with angle $\phi_{0}$ will leave the cap with angle $-\phi_{0}$. The net effect of the cap on a geodesic is thus determined by the rotation function, denoted by $\Delta \theta(\phi)$, that measures the angle, about the axis of revolution, through which a geodesic rotates before leaving the cap (figure 4).

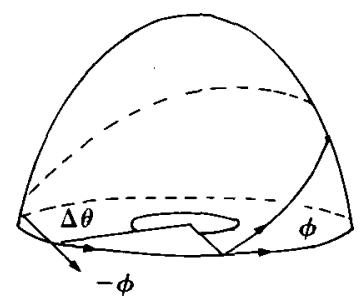

Figure 4. Rotation function $\Delta \theta$.

For a half-sphere, $\Delta \theta(\phi) \equiv \pi$. For a general cap, one uses the Clairaut Integral to solve the equations of motion and finds that along an orbit

$$
\frac{d \theta}{d l}=\mp \frac{c}{r(l) \sqrt{r^{2}(l)-c^{2}}}
$$

where $c=$ value of Clairaut Integral $=R \cos \phi_{0}$ [7]. The minus (plus) sign holds when the geodesic is ascending (descending) the cap. Integrating (14) and using the symmetry of the ascending and descending motions, the rotation function is given for $\phi \in(0, \pi / 2)$ by

$$
\Delta \theta(\phi)=2 c \int_{l_{\phi}}^{L} \frac{d l}{r(l) \sqrt{r^{2}(l)-c^{2}}},
$$

where $c=R \cos \phi$ and $r\left(l_{\phi}\right)=c$. The meridians are geodesics so that

$$
\begin{aligned}
\Delta \theta(\pi / 2) & =\pi \\
& =\lim _{\phi \rightarrow \pi / 2} \Delta \theta(\phi) .
\end{aligned}
$$

For $\phi \in(\pi / 2, \pi)$, we define $\Delta \theta$ using symmetry. A geodesic entering the cap at angle $\phi$ rotates counter-clockwise by the same amount that a geodesic entering at angle $(\pi-\phi)$ rotates clockwise. Hence we could define $\Delta \theta(\phi)=-\Delta \theta(\pi-\phi)$ for $\phi \in(\pi / 2, \pi)$. The resulting function would be discontinuous at $\phi=\pi / 2$. Instead, we define

$$
\Delta \theta(\phi)=2 \pi-\Delta \theta(\pi-\phi), \quad \phi \in(\pi / 2, \pi) .
$$

The resulting function is now continuous at $\phi=\pi / 2$.

Leмma 4.1. The rotation function $\Delta \theta(\phi)$, defined by equations (15-17), is $C^{\infty}$.

Proof. The geodesics cross the equator transversely. The implicit-function theorem, together with the smoothness of the geodesic flow, implies the result.

When we say the rotation function is given by (15), we mean (15) holds for $\phi \in(0, \pi / 2)$ and (16) and (17) give the values of $\Delta \theta(\phi)$ for $\phi \in[\pi / 2, \pi)$. 
To attach the cap smoothly to the surface, it may be necessary to shrink the cap. Such a process does not effect the rotation function.

Scaling Lemma 4.2. The rotation function is invariant under scalings of the cap.

Let $\tilde{r}(l), l \in[0, \tilde{L}]$, define a cap with rotation function $\Delta \tilde{\theta}(\phi)$. Then the function $r(l)=s \tilde{r}(l / s), l \in[0, s \tilde{L}]=[0, L]$, defines a rotation function $\Delta \theta(\phi)$ which satisfies $\Delta \theta(\phi)=\Delta \tilde{\theta}(\phi)$. To see this, draw the path of a geodesic on a cap and scale the picture.

\section{Focusing cap}

A cap satisfying the following properties is called a focusing cap.

(a) $(d / d l) r(l)>0$ for $l \in[0, L)$.

(b) On the equator, the curvature is zero.

(c) The equator is a geodesic.

(d) The rotation function is monotone decreasing,

$$
\frac{d}{d \phi} \Delta \theta(\phi)=\Delta \theta^{\prime}(\phi)<0 \quad \text { for } \phi \in(0, \pi) .
$$

Condition (b) allows us to attach the cap smoothly to the $K<0$ part of the surface. Condition (d) means that the smaller the angle of entry, the more a geodesic rotates before leaving the cap. Together with (c), it implies that a variation that is diverging when entering the cap will also be diverging when leaving the cap, which is the crux of Theorem 8.4.

Give the unit tangent space of the equator coordinates $(\theta, \phi), \theta \in[0,2 \pi), \phi \epsilon$ $[0,2 \pi)$. Let $\left(\theta^{\prime}, \phi^{\prime}\right)$ be the variation corresponding to the tangent vector $\bar{\xi}=$ $\theta^{\prime} X_{\theta}+\phi^{\prime} X_{\phi}$. The equator is a geodesic, so covariant differentiation simplifies, and the condition for a variation, entering the cap, to be divergent is $\phi^{\prime} / \theta^{\prime}<0$. The vectors in the variation are pointing away from one another. When the vectors leave the cap, the condition $\Delta \theta^{\prime}(\phi)<0$ insures that the variation is again diverging, since the relative order of the vectors in the variation has been preserved (figure 5).

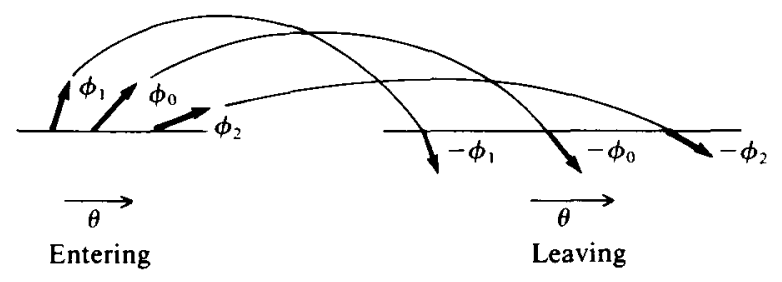

FIGURE 5. Diverging variation goes to diverging variation.

In $\S 9-11$, we show that, providing the equator remains a geodesic, we can weaken the other conditions on the cap and still produce positive entropy.

THEOREM 5.1. There exist focusing caps.

Proof. For a cap satisfying (a)-(c), one has $\Delta \theta^{\prime}(\phi)<0$ for small $\phi$ (Proposition A1.1). If $\Delta \theta^{\prime}(\phi)$ is not negative for all $\phi$, we produce a new cap with this property. 
We attach a cylinder of height $h$ to the base of the original cap. A geodesic entering the bottom of the cylinder with angle $\phi \in(0, \pi / 2)$ will leave at the top with the same angle haying rotated by an amount

$$
\Delta \theta_{c}(\phi)=\frac{h}{R} \cot \phi .
$$

For the cylinder, the smaller the angle of entry, the more the rotation. The combination of cap and cylinder is again a cap with rotation function $\Delta \tilde{\theta}=2 \Delta \theta_{c}+\Delta \theta$. Since $\Delta \theta(\phi)$ is $C^{1}$, its derivative, when positive, is bounded. Hence for $h$ sufficiently large, $\Delta \tilde{\theta}^{\prime}(\phi)<0$ for $\phi \in(0, \pi / 2)$. Since $\Delta \tilde{\theta}^{\prime}$ is continuous and by $(17)$, one then has $\Delta \tilde{\theta}^{\prime}(\phi)<0$ for all $\phi \in(0, \pi)$. The reason that adding a region of zero curvature can help produce exponential growth of Jacobi fields, i.e. non-zero exponents, is discussed in $\$ 9$.

The focusing cap we have produced is not necessarily smooth. In Appendix A.1, we generalize this argument to produce $C^{\infty}$ smooth focusing caps.

Burns and Gerber [6] give a different construction of focusing caps. They show that if the curvature of a cap satisfying (b), (c) is non-increasing,

$$
\frac{d}{d l} K(l) \leq 0, \quad l \in[0, L],
$$

then the rotation function is non-increasing,

$$
\frac{d}{d \phi} \Delta \theta(\phi) \leq 0, \quad \phi \in(0, \pi)
$$

If a geodesic starting with angle $\phi$ goes through a point at which $d K / d l(l)<0$, then $(d / d \phi) \Delta \theta(\phi)<0$.

\section{Effect of the cap on Jacobi fields}

We determine the value of a Jacobi field when it leaves a focusing cap (18) in terms of its value when it enters the cap.

Since Jacobi fields are parametrized using arc-length, we use the length coordinate $\rho$ rather than the angle coordinate $\theta$ to parametrize the equator. We get a new rotation function

$$
\Delta \rho(\phi)=R \Delta \theta(\phi)
$$

where $R$ is the radius of the equator. The quantity $-\Delta \rho^{\prime}(\phi) \sin \phi$ will come up often in our calculations, we denote it by

$$
h(\phi)=-\Delta \rho^{\prime}(\phi) \sin \phi, \quad \phi \in(0, \pi),
$$

and since $\Delta \rho^{\prime}(\phi)<0$, we have $h(\phi)>0$. Under scalings of the cap, as in Lemma $4.2, \Delta \rho^{\prime}(\phi)$ changes by the scale factor, but does not change sign.

Proposition 6.1. A Jacobi field that has value $\left(J, J^{\prime}\right)$, when entering the cap at $(\rho, \phi)$, will have value $\left(\tilde{J}, \tilde{J}^{\prime}\right)$ when leaving the cap at $(\rho+\Delta \rho(\phi)(\bmod 2 \pi R),-\phi)$ with

$$
\left(\tilde{J}, \tilde{J}^{\prime}\right)=-\left(J+J^{\prime} h(\phi), J^{\prime}\right) \text {. }
$$

Under the identification of $\left(J, J^{\prime}\right)$ with $-\left(J, J^{\prime}\right)$, the map (21) is shown in figure 6. 

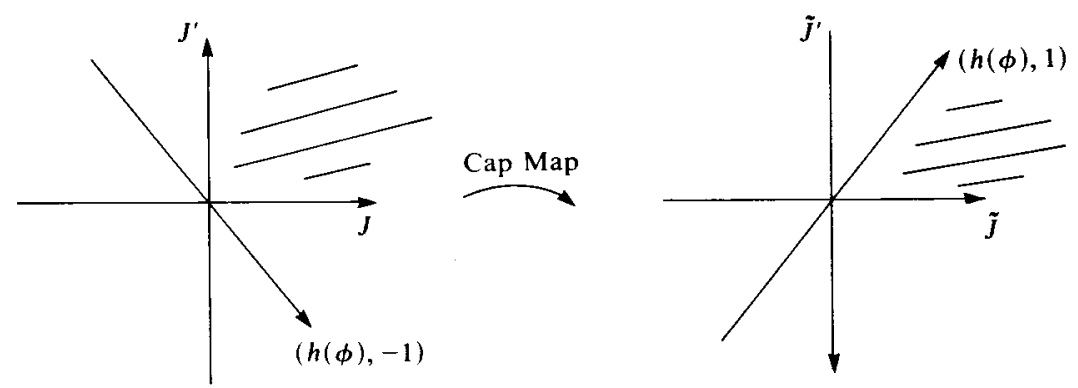

FIGURE 6. Jacobi field: entering and leaving cap.

Remark 6.2. A focusing cap has conjugate points. By (21), if $J=0$ and $J^{\prime}=1$, then $\tilde{J}<0$. Thus $J$ must vanish for a second time while it is in the cap.

To prove Proposition 6.1, first project the Jacobi field onto a variation based on the equator. Let $\mathscr{S}=\{(\rho, \phi): \rho \in[0,2 \pi R), \phi \in(0, \pi)\}$ be the set of geodesics entering the cap. These coordinates induce a basis $\left\{X_{\rho}, X_{\phi}\right\}$ for $T_{(\rho, \phi)} \mathscr{Y}$. At $(\rho, \phi)$, there is also the orthonormal basis $\left\{V, V^{\perp}, \Phi\right\}$ for $T_{(\rho, \phi)}(S M)(\S 2)$. Let $\xi$ be the vector corresponding to the Jacobi field $\left(J, J^{\prime}\right)$.

$$
\xi=J V^{\perp}+J^{\prime} \Phi
$$

Project $\xi$ in the $V$ direction to get a vector

$$
\bar{\xi}=\rho^{\prime} X_{\rho}+\phi^{\prime} X_{\phi} \in T_{(\rho, \phi)} \mathscr{I} .
$$

Projection Lemma 6.3. The relation between the $\left(J, J^{\prime}\right)$ and $\left(\rho^{\prime}, \phi^{\prime}\right)$ coordinates is

so that

$$
\rho^{\prime}=-\frac{J}{\sin \phi}, \quad \phi^{\prime}=J^{\prime}
$$

$$
\bar{\xi}=-\frac{J}{\sin \phi} X_{\phi}+J^{\prime} X_{\phi}
$$

Proof. Since $X_{\phi}$ and $\Phi$ are both unit vectors in the fiber direction,

$$
X_{\phi}=\Phi \text {. }
$$

The equator is a geodesic, so $X_{\rho}$ lies in the horizontal subspace spanned by $\left\{V, V^{\perp}\right\}$,

$$
X_{\rho}=(\cos \phi) V+(-\sin \phi) V^{\perp} \text {. }
$$

Thus the projection of $V^{\perp}$, in the $V$ direction, onto $X_{\rho}$ is

$$
V^{\perp} \mapsto-\frac{1}{\sin \phi} X_{\rho}
$$

The negative sign results from differing orientation in the definition of $V^{\perp}$ and $X_{\rho}$. Combining (26)-(27) proves the result.

Proof of Proposition 6.1. (i) Project the $\left(J, J^{\prime}\right)$ variation onto the equator to get a $\left(\rho^{\prime}, \phi^{\prime}\right)$ variation $\bar{\xi}$,

$$
\bar{\xi}=\rho^{\prime} X_{\rho}+\phi^{\prime} X_{\phi}=-\frac{J}{\sin \phi} X_{\rho}+J^{\prime} X_{\phi}
$$


(ii) Under the geodesic flow the incoming variation $\left(\rho^{\prime}, \phi^{\prime}\right)$ at the point $(\rho, \phi)$ gets sent to an outgoing variation $\left(\tilde{\rho}^{\prime}, \tilde{\phi}^{\prime}\right)$ at the point $(\tilde{\rho}, \tilde{\phi})=$ $(\rho+\Delta \rho(\phi)(\bmod 2 \pi R),-\phi)$ with

$$
\left(\tilde{\rho}^{\prime}, \tilde{\phi}^{\prime}\right)=\left(\rho^{\prime}+\Delta \rho^{\prime}(\phi) \phi^{\prime},-\phi^{\prime}\right) \text {. }
$$

(iii) Project the outgoing $\left(\tilde{\rho}^{\prime}, \tilde{\phi}^{\prime}\right)$ variation at $(\tilde{\rho}, \tilde{\phi})$ to the outgoing Jacobi field $\left(\tilde{J}, \tilde{J}^{\prime}\right)$

$$
\tilde{J}^{\prime}=\tilde{\phi}^{\prime}, \quad \tilde{J}=\tilde{\rho}^{\prime} \sin |\tilde{\phi}|=\tilde{\rho}^{\prime} \sin \phi .
$$

For the outgoing variation, the vectors $X_{\rho}$ and $V^{\perp}$ are oriented the same way, so there is no minus sign. Combining steps (i), (ii) and (iii) gives

$$
\begin{aligned}
\left(J, J^{\prime}\right) \rightarrow\left(\rho^{\prime}, \phi^{\prime}\right) & =\left(-\frac{J}{\sin \phi^{\prime}} J^{\prime}\right) \\
\rightarrow\left(\tilde{\rho}^{\prime}, \tilde{\theta}^{\prime}\right) & =\left(-\frac{J}{\sin \phi}+\Delta \rho^{\prime}(\phi) J^{\prime},-J^{\prime}\right) \\
\rightarrow\left(\tilde{J}, \tilde{J}^{\prime}\right) & =\left(-J+\Delta \rho^{\prime}(\phi) J^{\prime} \sin \phi,-J^{\prime}\right) \\
& =-\left(J+h(\phi) J^{\prime}, J^{\prime}\right) .
\end{aligned}
$$

\section{Attaching the cap}

To produce our metric on the sphere (or on surfaces of genus $g \geq 1$ ), we smoothly attach a focusing cap (18) to the punctured surface of curvature $K \equiv-1$. Outside the caps, the curvature will satisfy $K<0$.

Take a focusing cap defined by a $C^{\infty}$ function $r_{1}(l), l \in\left[0, L_{1}\right]$, for which

$$
r_{1}(l)=r_{1}\left(L_{1}\right)+\frac{r_{1}^{(2 k+1)}\left(L_{1}\right)}{(2 k+1) !}\left(l-L_{1}\right)^{2 k+1}+O\left(\left(l-L_{1}\right)^{2 k+2}\right),
$$

with $k \geq 1$.

The embedded $K \equiv-1$ cusp is rotationally symmetric. We choose the parallel on the cusp at which we want the $K \equiv-1$ region to stop and assign it an arbitrary arc-length coordinate $L_{2}$ with $L_{2}>L_{1}$. After our construction, $L_{2}$ will be the distance of this parallel from the top of the cap. The metric on the cusp is

$$
d s^{2}=d l^{2}+r_{2}^{2}(l) d \theta^{2}, \quad l \in\left(-\infty, L_{3}\right],
$$

where $L_{3}>L_{2}$ and $L_{3}$ corresponds to the base of the cusp, i.e. the parallel at which the cusp ceases to be embeddable. The curvature on the cusp is

$$
K(l)=-r_{2}^{\prime \prime}(l) / r_{2}(l)=-1 .
$$

The radius at the equator of the cap must satisfy $r_{1}\left(L_{1}\right)<r_{2}\left(L_{2}\right)$, but this can always be achieved by shrinking the cap. Such scalings do not effect the essential properties of the cap (Lemma 4.2).

We now use a partition of unity function on the interval $\left[L_{1}, L_{2}\right]$ to splice together $r_{1}$ and $r_{2}$ (see Appendix A.2 for details). To insure that $K<0$ in this transitional region, $r_{1}(l)$ must behave like an odd power of $\left(l-L_{1}\right)$ for $l$ near $L_{1}$.

\section{Positive entropy}

For the surfaces constructed in $\S 7$, we use Wojtkowski's method of invariant cones to show that almost every point $x \in S M$ has a non-zero Lyapunov exponent. 
THEOREM 8.1. Let $M$ be a compact surface with $C^{\infty}$ Riemannian metric that contains focusing caps such that outside the caps the curvature satisfies $K<0$. Then the Lyapunov exponents of the geodesic flow $g_{t}$ on SM are almost everywhere non-zero.

We have shown how to produce, on a compact surface of arbitrary genus, a metric satisfying these assumptions. Thus this theorem, together with Pesin's formula (3), implies Theorem 1.

For each $x \in S M$, we will define a cone of tangent vectors $\mathscr{C}(x)$ contained in the perpendicular subspace of $T_{x} S M(\S 2)$. The family of perpendicular subspaces is invariant under the linearized flow $D g_{t}$. We examine the time one flow $g_{1}$ and its linearization $D g_{1}$.

The cone-field is said to be invariant if for almost every $x$,

$$
D g_{1}(x)(\mathscr{C}(x)) \subseteq \mathscr{C}\left(g_{1}(x)\right) \text {. }
$$

The cone-field is strictly invariant if it is invariant and if for almost every $x$ both boundary lines of $D g_{1}(x)(\mathscr{C}(x))$ are contained strictly inside $\mathscr{C}\left(g_{1}(x)\right)$. Finally, the cone-field is eventually strictly invariant if it is invariant and if for almost every $x$ there is an $n(x)$ such that both boundary lines of $D g_{1}^{n(x)}(x)(\mathscr{C}(x))$ are contained strictly inside $\mathscr{C}\left(g_{1}^{n(x)}(x)\right)$. The cone-field is measurable if $\mathscr{C}(x)$ depends measurably on $x$.

THEOREM 8.2. If there exists a measurable cone-field $\{\mathscr{C}(x)\}, x \in S M$, that is eventually strictly invariant under the time one map, then almost every point has a non-zero Lyapunov exponent for the geodesic flow.

This theorem follows immediately from Theorem 2.2 [17] once we express the map $D g_{1}$ in terms of the basis $\left\{V, V^{\perp}, \Phi\right\}$.

The important thing to check when defining the cone-field is that it is strictly invariant under returns to the cap. If this is so, one can easily define the cone-field over the rest of $S M$. For each cap, let

$$
\begin{aligned}
\mathscr{S}_{i} & =\{\text { equator }\} \times\{\text { vectors pointing into cap }\} \\
& =\{(\rho, \phi): \rho \in[0,2 \pi R), \phi \in(0, \pi)\} .
\end{aligned}
$$

We set $\mathscr{S}$ equal to the union of these disjoint sets.

For $x \in \mathscr{S}$, we define

$$
\mathscr{C}(x)=\left\{J V^{\perp}+J^{\prime} \Phi \in T_{x} S M: 0 \leq J^{\prime} / J \leq+\infty\right\} .
$$

This cone corresponds to the diverging Jacobi fields based at $x$. By $J^{\prime} / J=+\infty$ we mean $J=0$.

When $x$ leaves the cap, equation (21) gives that if $\left(J, J^{\prime}\right) \in \mathscr{C}(x)$, then $0 \leq \tilde{J}^{\prime} / \tilde{J} \leq$ $h(\phi)$, where $\phi$ is the angle with which $x$ enters the cap.

Outside the cap, the evolution of $J^{\prime} / J$ is determined by the Riccati equation (8). In curvature $K \leq 0$, a diverging Jacobi field stays diverging.

Lemma 8.3. Let $J^{\prime} / J(t)$ be a solution of the Riccati equation with $K(t) \leq 0$ for $t \geq 0$. (a) If $0 \leq J^{\prime} / J(0) \leq+\infty$, then $0 \leq J^{\prime} / J(t)<+\infty$ for $t>0$. 
(b) If $J^{\prime} / J(0)=0$ and there exists a $t^{*}>0$ such that $K\left(t^{*}\right)<0$, then $J^{\prime} / J\left(t^{*}\right)>0$.

(c) (Ordering Property) Let $J_{1}^{\prime} / J_{1}$ be a second solution of the same Riccati equation. If $0 \leq J_{1}^{\prime} / J_{1}(0)<J^{\prime} / J(0) \leq+\infty$ then $0 \leq J_{1}^{\prime} / J_{1}(t)<J^{\prime} / J(t)<+\infty$ for $t>0$.

For our surfaces $M$, every point goes through strictly negative curvature between returns to the cap. Hence if $x$ returns to the cap at time $\tau_{1}$ at the point $x_{1}$, we have that $D g_{\tau_{1}}(x)(\mathscr{C}(x))$ is contained strictly inside $\mathscr{C}\left(x_{1}\right)$.

To formalize the argument, we define the cone-field for all of $S M$. If $x$ has basepoint outside the caps, then we define $\mathscr{C}(x)$ as in (32). If $x^{\prime}$ has basepoint in a cap, then by Proposition 3.1 there is an $x \in \mathscr{S}$ that will hit $x^{\prime}$ : for some $t>0$, $g_{t} x=x^{\prime}$. Choose the $x \in \mathscr{P}$ for which $t$ is minimal. We define

$$
\mathscr{C}\left(x^{\prime}\right)=D g_{t}(x)(\mathscr{C}(x)) \text {. }
$$

THEOREM 8.4. On a surface $M$ satisfying the assumptions of Theorem 8.1, the cone-field defined by (32) and (33) is almost everywhere eventually strictly invariant.

This together with Theorem 8.2 proves Theorem 8.1. Note that the crucial part in making the cone-field strictly invariant was that geodesics went through some point of strictly negative curvature while outside the caps (Lemma 8.3b). Hence we have the following more general result.

THEOREM 8.5. Let $M$ be a compact surface with $C^{\infty}$ Riemannian metric that contains focusing caps and such that

(a) outside the caps, the curvature satisfies $K \leq 0$,

(b) almost every geodesic, while outside the caps, goes through a point of strictly negative curvature.

Then the Lyapunov exponents of the geodesic flow are non-zero almost everywhere.

Remark 8.6. The example of flat-torus with focusing cap (figure 3) will satisfy conditions (a) and (b) providing we attach the cap to the $K \equiv 0$ region by a collar of strictly negative curvature.

We thank the referee for suggesting that we use the time one map to prove Theorem 8.1. This approach is simpler than our original proof in [8]. There we proved that almost every geodesic enters the caps infinitely often. Then, using the cone-field (32), we showed that the Lyapunov exponents for the return map to $\mathscr{S}$ are positive almost everywhere, which implies Theorem 8.1 .

\section{Semi-focusing cap}

A cap satisfying (18a-c) will have $\Delta \theta^{\prime}(\phi)<0$ for small $\phi$, but not necessarily for all $\phi$. If there exists a $\phi$ for which $\Delta \theta^{\prime}(\phi)>0$, we call the cap semi-focusing. By the cone-field argument, Theorem 8.5 will hold for semi-focusing caps as well, provided we are careful in how we attach the cap to the $K \leq 0$ region.

For $x=(\rho, \phi) \in \mathscr{Y}$, we define the cone $\mathscr{C}(\rho, \phi)$ by

$$
\mathscr{C}(\rho, \phi)=\left\{\begin{array}{c}
\text { Jacobi fields based at }(\rho, \phi) \text { that are diverging both } \\
\text { when they enter and when they leave the cap. }
\end{array}\right\}
$$


For $\Delta \rho^{\prime}(\phi)=R \Delta \theta^{\prime}(\phi)>0,(21)$ implies that this cone is given by

$$
\mathscr{C}(\rho, \phi)=\left\{\left(J(0), J^{\prime}(0)\right): \frac{J^{\prime}}{J}(0) \in\left[0, \frac{1}{\Delta \rho^{\prime}(\phi) \sin \phi}\right]\right\} .
$$

For $\phi$ with $\Delta \rho^{\prime}(\phi) \leq 0, \mathscr{C}(\rho, \phi)$ is given by (32). These cones are not necessarily invariant under returns to $\mathscr{S}$.

To be specific, assume $x=(\rho, \phi) \in \mathscr{S}$ leaves the cap at time $\tilde{\tau}_{0}$ and returns at time $\tau_{1}$ with angle $\phi^{*}$, where $\Delta \rho^{\prime}\left(\phi^{*}\right)>0$. If $\left(J(0), J^{\prime}(0)\right) \in \mathscr{C}(\rho, \phi)$, then $J^{\prime} / J\left(\tilde{\tau}_{0}\right) \in$ $[0, M(x)]$, where $M(x)=1 / h(\phi)$ (see (20)) if $\Delta \rho^{\prime}(\phi)<0$ and $M(x)=+\infty$ otherwise.

Let $u(t ; x)$ be the Riccati solution based at $x$ satisfying the condition $u\left(\tilde{\tau}_{0} ; x\right)=$ $M(x)$. For invariance to hold, it is necessary, and by the ordering property (Lemma $8.3 c)$ also sufficient, that

$$
u\left(\tau_{1} ; x\right) \leq \frac{1}{\Delta \rho^{\prime}\left(\phi^{*}\right) \sin \phi^{*}} .
$$

Since $\Delta \rho(\phi)$ is $C^{1}$, its derivative, when positive, is bounded. Hence the top of the cones $\mathscr{C}\left(\rho, \phi^{*}\right)$ are bounded away from zero: if

$$
m=\inf _{\left\{\phi^{*}: \Delta \rho^{\prime}\left(\phi^{*}\right)>0\right\}} \frac{1}{\Delta \rho^{\prime}\left(\phi^{*}\right) \sin \phi^{*}},
$$

then $m>0$. By the properties of the Riccati equation, $u\left(\tau_{1} ; x\right)$ will be less than $m$ if, for a suitably long period of time immediately preceding $\tau_{1}$, the curvature $K(t) \leq 0$ is sufficiently close to zero (suitable, sufficient depending on $m$ ). Thus $u\left(\tau_{1} ; x\right)<m$ will hold for all geodesics returning to the cap if next to the equator, in the $K \leq 0$ region, we attach a strip of suitable width on which the curvature is uniformly close to zero. This condition is similar to the type of requirements Wojtkowski [18] places on his billiard tables. Once $u\left(\tau_{1} ; x\right)<m$ holds for all $x \in \mathscr{Y}$, we can define an eventually strictly invariant cone-field on all of $S M$.

This discussion also shows why attaching a cylinder with $K \equiv 0$ to the base of a semi-focusing cap (Theorem 5.1) produces a focusing cap: one for which any diverging Jacobi field that enters the cap is again diverging when it leaves. If $J^{\prime} / J \in[0,+\infty]$ at the base of the cylinder then, providing the cylinder has height $h>1 / m$, the Jacobi field satisfies $J^{\prime} / J<m$ when it reaches the semi-focusing cap. By (34), (35) and (37), this insures that the Jacobi field will be diverging when it leaves the semi-focusing cap, and it then stays diverging as it descends the cylinder.

\section{Non-focusing caps}

For a flat torus, the phase space of the geodesic flow decomposes into invariant tori. Our focusing cap can be considered a perturbation of the flat metric. Bangert's [4] result implies that, under this perturbation, all the invariant tori have broken up. Clearly this result is necessary if the geodesic flow is to be ergodic.

Consider the flat torus with focusing cap as the end product of a one-parameter family of metrics on the torus that start with the flat metric and for which the intermediate metrics have caps given by (38). How far in this family must we go until the entropy first becomes positive? Unfortunately, the cone-field technique fails to prove positive entropy for any of the intermediate metrics. 
We take a cap for which the curvature on the equator is zero, but the equator is not a geodesic. Such a cap is defined by

$$
r^{\prime}(l)>0, \quad l \in[0, L], \text { and } r^{\prime \prime}(L)=0 .
$$

The map from $\left(J, J^{\prime}\right) \mapsto\left(\tilde{J}, \tilde{J}^{\prime}\right)$ is no longer given by (21). Outside the cap, the curvature should still satisfy $K \leq 0$. For such a surface, the cones $\mathscr{C}(x), x \in \mathscr{S}$, defined by (34) will not be invariant under returns to $\mathscr{S}$.

By the Clairaut Integral, a geodesic that is tangent to the equator will immediately leave the cap and re-enter the $K \leq 0$ region. A diverging Jacobi field on this geodesic stays diverging the 'whole time' it is in the cap, so is diverging when it leaves the cap and then continues to diverge until its next return.

Any metric on the sphere has conjugate points [11]. To illustrate the obstruction to an invariant cone-field, suppose that the geodesic starting at $\left(\rho, \phi_{*}\right) \in \mathscr{S}$ has a conjugate point in the cap.

A diverging Jacobi field based at $\left(\rho, \phi_{*}\right)$ will again be diverging when it leaves the cap, but now having focused once while in the cap. By continuity, there exist angles between 0 and $\phi_{*}$ for which the diverging variations start to converge while in the cap, but not enough to focus. They will then be converging when they leave the cap and stay converging until their next return.

This behaviour implies that for the cones given by (34), for any $k \in(0, \infty)$ there is an angle $\phi_{k} \in\left(0, \phi_{*}\right)$ such that

$$
\mathscr{C}\left(\rho, \phi_{k}\right)=\left\{\left(J, J^{\prime}\right): \frac{J^{\prime}}{J} \in[k,+\infty]\right\},
$$

(compare with (35)). For geodesics that return to the cap at $\left(\rho, \phi_{k}\right) \in \mathscr{S}$, with $k$ large, the cones will clearly not be invariant.

Although one may try to define a different cone-family, we feel that the continuity factor will always prevent it from being almost everywhere invariant. Thus new techniques are needed to determine the Lyapunov exponents for a surface with caps given by (38).

\section{Positive entropy but non-ergodic surface}

Take a cap in the shape of a light-bulb (figure 7). By the properties of the Clairaut Integral, there will be a set of geodesics, of positive measure, that start inside the cap but never leave it. The motion of these geodesics is integrable. The geodesics that enter the cap will leave. Choose the cap so that the rotation function, as measured from the neck of the light bulb, satisfies $\Delta \theta^{\prime}(\phi)<0$ for $\phi \in(0, \pi)$. That such a cap exists can be shown by modifying the proof of Theorem 5.1.

If we now attach such caps to a surface satisfying the assumptions of Theorem 8.5 , the resulting system is not ergodic. The geodesics that start either outside the caps or inside the caps but leave the caps form an invariant set of positive measure. By the cone argument, we can show that almost every point in this set has a non-zero Lyapunov exponent, and so the entropy is positive.

Here the phase space decomposes into an integrable component, consisting of the orbits that stay in the cap, and a stochastic component, consisting of the orbits that have positive exponents. 


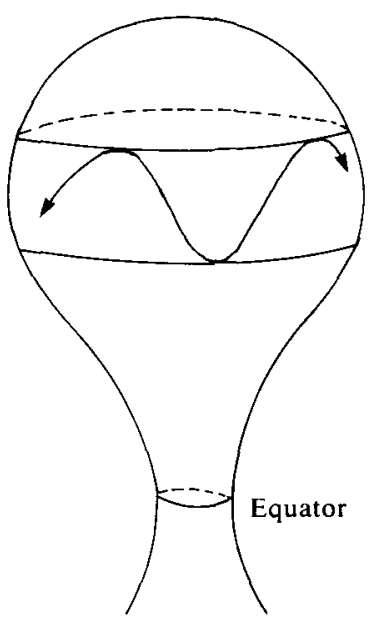

FIGURE 7. Light-bulb cap.

12. Appendix

A.1. Construction of focusing cap

The crucial part in building a cap with the desired properties is controlling the behaviour of the rotation function for small $\phi$. Let the equator be a geodesic on which the curvature vanishes to a finite order, and let $(d / d l) r(l)>0$ for $l$ near $L$. For such a cap,

$$
r(l)=r(L)+\frac{r^{(n)}(L)}{n !}(l-L)^{n}+O\left((l-L)^{n+1}\right),
$$

where $l \in[0, L], n \geq 3$ and $r^{(n)}(L)(l-L)^{n}<0$.

Proposition A1.1. (1) For a cap given by (A1.1),

$$
\lim _{\phi \rightarrow 0} \Delta \theta(\phi)=+\infty \text {. }
$$

(2) For small $\phi$,

$$
\Delta \theta^{\prime}(\phi)<0 \text {. }
$$

Proof. (1) Let $\gamma\left(t, \theta_{0}, \phi\right)$ be the geodesic that at time $t=0$ starts at the point $\theta_{0}$ on the equator with angle $\phi>0$. After time $t$, the distance between this geodesic and the equator is given by

$$
d\left(\gamma\left(t, \theta_{0}, \phi\right)\right)=J(t) \phi+O\left(\phi^{2}\right) .
$$

$J(t)$ is the solution of the Jacobi equation based on the equator at $\left(\theta_{0}, \phi=0\right)$ with initial conditions $J(0)=0$ and $J^{\prime}(0)=1$. Since $K(t) \equiv 0$ along the equator, we have that $J(t)=t$. Hence for a given $T>0$, there exists a $\delta>0$ such that for $0<\phi<\delta$, one has

$$
d\left(\gamma\left(t, \theta_{0}, \phi\right)\right)>0 \quad \text { for } 0<t \leq T .
$$

The geodesic has not yet crossed the equator. For such $\phi, \Delta \theta(\phi) \geq T / R$.

(2) A sufficient condition for the limit in (A1.2) to be obtained monotonically is 
stated in (A1.9). This condition, satisfied by all functions of the form (A1.1), is given in terms of $(r, \theta)$, rather than $(l, \theta)$, variables.

When $r(l)$ is monotone, we can realize our cap by revolving a function $z=f(r)$ about the $z$-axis. In $(r, \theta)$ coordinates, the surface is given by $x=r \cos \theta, y=r \sin \theta$, $z=f(r)$. Let

$$
g(r)=\sqrt{1+f^{\prime 2}(r)}
$$

then the metric is given by

$$
d s^{2}=g^{2}(r) d r^{2}+r^{2} d \theta^{2}
$$

Since $l(r)=\int_{0}^{r} g(s) d s$, we have

$$
d l / d r=g(r) .
$$

In these variables, the rotation function (15) becomes

$$
\Delta \theta(\phi)=2 c \int_{c}^{R} \frac{g(r)}{r \sqrt{r^{2}-c^{2}}} d r, \quad c=R \cos \phi .
$$

LEMMA A1.2. Given a $\delta>0$, there exists an $r_{0} \in(0, R)$ such that for all $c \in\left[r_{0}, R\right)$,

$$
\frac{d}{d c} \Delta \theta(c)>\frac{2 c}{(R-c)} \int_{c}^{R}\left\{g^{\prime}(r)(R-r)-(1+\delta) \frac{g(r)}{2}\right\} \frac{d r}{r \sqrt{r^{2}-c^{2}}} .
$$

The value $r_{0}(\delta)$ is independent of $g$.

This lemma, whose proof appears at the end of the section, implies that if

$$
\lim _{r \rightarrow R}\left\{g^{\prime}(r)(R-r)-\frac{g(r)}{2}\right\}>0
$$

then for $c$ sufficiently close to $R$, we have $(d / d c) \Delta \theta(c)>0$. This implies that

$$
\frac{d}{d \phi} \Delta \theta(\phi)=\frac{d}{d c} \Delta \theta(\phi) \frac{d c}{d \phi}
$$

is negative for $\phi$ close to zero since $d c / d \phi=-R \sin \phi$.

Inverting (A1.1) gives

$$
(L-l)=k_{1}(R-r)^{1 / n}+O\left((R-r)^{2 / n}\right), \quad k_{1}>0
$$

so that for the cap under consideration,

$$
g(r)=\frac{d l}{d r}=\frac{k_{2}}{(R-r)^{(n-1) / n}}+O\left(\frac{1}{(R-r)^{(n-2) / n}}\right), \quad k_{2}=\frac{k_{1}}{n} .
$$

Omitting higher order terms,

$$
\begin{aligned}
g^{\prime}(r)(R-r)-\frac{1}{2} g(r) & =\frac{k_{2}}{(R-r)^{(n-1) / n}}\left\{\frac{n-1}{n}-\frac{1}{2}\right\} \\
& >0, \quad \text { for } n>2, r<R,
\end{aligned}
$$

which, by the preceding comments, proves (A1:3).

Proof of Theorem 5.1. Let $r(l), l \in[0, L]$, define a cap with $(d / d l) r(l)>0$ for $l \in[0, L)$ that satisfies (A1.1) and is $C^{\infty}$. For simplicity let

$$
r(l)=r(L)+a_{n}(l-L)^{n},
$$


$n \geq 3$, for $l$ near $L$. If this cap does not satisfy $\Delta \theta^{\prime}(\phi)<0$ for all $\phi \in(0, \pi)$, we will produce a cap with this property by changing $r(l)$ for $r$ near $R$. This change will result in a cap which is nearly flat, i.e. $d r / d l$ is close to zero, for a long interval next to the equator.

For $r$ near $R$, the function $g(r)=(d l / d r)(r)$ is given by

$$
g(r)=\frac{k}{(R-r)^{(n-1) / n}},
$$

where $k=k\left(a_{n}, n\right)>0$. By (A1.3), for small $\phi, \Delta \theta^{\prime}(\phi)<0$. To be specific, choose $\delta>0$ such that

$$
\lim _{r \rightarrow R}\left\{g^{\prime}(r)(R-r)-(1+\delta) \frac{g(r)}{2}\right\}>0 .
$$

Then using Lemma (A1.2), pick $r_{1} \in(0, R)$ such that

$$
\frac{d}{d c} \Delta \theta(c)>\frac{2 c}{R-c} \int_{c}^{R}\left\{g^{\prime}(r)(R-c)-(1+\delta) \frac{g(r)}{2}\right\} \frac{d r}{r \sqrt{r^{2}-c^{2}}}>0,
$$

for all $c \in\left[r_{1}, R\right)$. Denote by $\phi_{r}$ the angle satisfying $r=R \cos \phi_{r}$. A geodesic starting at the equator with angle $\phi_{r}$ will turn around at radius $r$. Equation (A1.14) implies that

$$
\Delta \theta^{\prime}(\phi)<0, \quad \phi \in\left(0, \phi_{r_{1}}\right] \cup\left[\pi-\phi_{r_{1}}, \pi\right) .
$$

For $\phi \in\left[\phi_{r_{1}}, \pi-\phi_{r_{1}}\right]$, it remains to show that $(d / d \phi) \Delta \theta(\phi)$ is negative. For such $\phi$, write the rotation function in two parts; the rotation between $R$ and $r_{2}$, and the rotation between $r_{2}$ and $c$, where $r_{2}$ is an arbitrary radius satisfying $r_{1}<r_{2}<R$.

$$
\Delta \theta(\phi)=\Delta \theta_{1}(\phi)+\Delta \theta_{2}(\phi)
$$

where

$$
\begin{aligned}
& \Delta \theta_{1}(\phi)=2 c \int_{r_{2}}^{R} \frac{g(r)}{r \sqrt{r^{2}-c^{2}}} d r, \quad c=R \cos \phi<r_{2} \\
& \Delta \theta_{2}(\phi)=2 c \int_{c}^{r_{2}} \frac{g(r)}{r \sqrt{r^{2}-c^{2}}} d r .
\end{aligned}
$$

For $\phi \in\left(\phi_{r_{2}}, \pi-\phi_{r_{2}}\right)$, the geodesics cross the parallel $r=r_{2}$ transversely, so $\Delta \theta_{2}(\phi)$ is a $C^{1}$-function on $\left(\phi_{r_{2}}, \pi-\phi_{r_{2}}\right)$. Hence for $\phi \in\left[\phi_{r_{1}}, \pi-\phi_{r_{1}}\right] \subset\left(\phi_{r_{2}}, \pi-\phi_{r_{2}}\right)$, there exists a constant $c_{2}>0$ such that

$$
\Delta \theta_{2}^{\prime}(\phi) \leq c_{2}
$$

Since $c<r_{2}$, we can find the derivative of $\Delta \theta_{1}$ directly. For $\phi \in\left[\phi_{r_{1}}, \pi-\phi_{r_{1}}\right]$,

$$
\begin{aligned}
\frac{d}{d \phi} \Delta \theta_{1}(\phi) & =-2 R \sin \phi \int_{r_{2}}^{R} \frac{g(r) r}{\left(r^{2}-c^{2}\right)^{3 / 2}} d r \\
& \leq\left(-2 R \sin \phi_{r_{2}}\right) \frac{r_{2}}{R^{3}} \int_{r_{2}}^{R} g(r) d r \\
& \leq-c_{1} \int_{r_{2}}^{R} g(r) d r,
\end{aligned}
$$

where $c_{1}=\left(2 r_{2} / R^{2}\right) \sin \phi_{r_{2}}=c_{1}\left(r_{2}, R\right)$ is independent of $g$. Combining (A1.17)- 
(A1.18) gives,

$$
\Delta \theta^{\prime}(\phi) \leq-c_{1} \int_{r_{2}}^{R} g(r) d r+c_{2}, \text { for } \phi \in\left[\phi_{r_{1}}, \pi-\phi_{r_{1}}\right],
$$

which will be negative providing

$$
l(R)-l\left(r_{2}\right)=\int_{r_{2}}^{R} g(r) d r>\frac{c_{2}}{c_{1}} .
$$

If (A1.20) is not satisfied, we can change $g$ in a small neighborhood of $R$ so that it will become satisfied. We must be careful that after this change, (A1.15) remains valid. Let

$$
g_{1}(r)=\frac{k_{1}}{(R-r)^{\left(n_{1}-1\right) / n_{1}}},
$$

where $n_{1}>n$ and $k_{1}$ are chosen so that

$$
g_{1}(r)>g(r), \quad r \in\left[r_{2}, R\right),
$$

and

$$
\int_{\left(r_{2}+R\right) / 2}^{R} g_{1}(r) d r>\frac{c_{2}}{c_{1}} .
$$

Let $p(r)$ be a $C^{\infty}$ partition of unity function satisfying $p(r) \equiv 0$ for $r \leq r_{2}, p^{\prime}(r)>0$ for $r \in\left[r_{2},\left(r_{2}+R\right) / 2\right]$ and $p(r) \equiv 1$ for $r \geq\left(r_{2}+R\right) / 2$. Define a new cap with rotation function $\Delta \tilde{\theta}$ by the function

$$
\tilde{g}(r)=(1-p(r)) g(r)+p(r) g_{1}(r) .
$$

Then for $c \in\left[r_{1}, R\right)$,

$$
\frac{d}{d c} \Delta \tilde{\theta}(c) \geq \frac{2 c}{R-c} \int_{c}^{R}\left\{\tilde{g}^{\prime}(r)(R-r)-(1+\delta) \frac{\tilde{g}(r)}{2}\right\} \frac{d r}{r \sqrt{r^{2}-c^{2}}},
$$

by Lemma (A1.2) and choice of $r_{1}$,

$$
\begin{aligned}
\geq & \frac{2 c}{R-c} \int_{c}^{R}\left[p^{\prime}\left(g_{1}(r)-g(r)\right)(R-r)+(1-p)\right. \\
& \times\left\{g^{\prime}(r)(R-r)-\frac{(1+\delta)}{2} g(r)\right\} \\
& \left.+p\left\{g_{1}^{\prime}(r)(R-r)-\frac{(1+\delta)}{2} g_{1}(r)\right\}\right] \frac{d r}{r \sqrt{r^{2}-c^{2}}}>0,
\end{aligned}
$$

by (A1.14), (A1.21). Therefore $\Delta \tilde{\theta}^{\prime}(\phi)<0$ for $\phi \in\left\{\left(0, \phi_{r_{1}}\right] \cup\left[\pi-\phi_{r_{1}}, \pi\right)\right\}$. For $\phi \in$ $\left[\phi_{r_{1}}, \pi-\phi_{r_{1}}\right]$, the same estimates as before combined with (A1.22) now insure that $\Delta \tilde{\theta}^{\prime}(\phi)<0$.

Proof of Lemma A1.2. To differentiate (A1.7) with respect to $c$, make the change of variables

$$
r=c+v(R-c), \quad d r=(R-c) d v
$$

which gives

$$
\Delta \theta(c)=2 c \int_{0}^{1} \frac{g(c+v(R-c))(R-c)}{(c+v(R-c)) \sqrt{2 c+v(R-c)}} \frac{d v}{\sqrt{v(R-c)}}
$$


Set

$$
\alpha=\alpha(c, v)=c+v(R-c), \quad \beta=\beta(c, v)=\sqrt{2 c+v(R-c)},
$$

so that

$$
\Delta \theta(c)=2 c \int_{0}^{1} \frac{g(\alpha(c, v)) \sqrt{R-c}}{\alpha(c, v) \beta(c, v)} \frac{d v}{\sqrt{v}} .
$$

Differentiation gives,

$$
\begin{aligned}
\frac{d}{d c} \Delta \theta(c)= & 2 \int_{0}^{1} \frac{g(\alpha) \sqrt{R-c} \frac{d v}{\sqrt{v}}}{\alpha \beta} \\
& +2 c \int_{0}^{1}\left(\frac{g^{\prime}(\alpha) \alpha^{\prime} \sqrt{R-c}-g(\alpha) \frac{1}{2 \sqrt{R-c}}}{\alpha \beta}-g(\alpha) \sqrt{R-c}\left\{\frac{\alpha^{\prime} \beta+\alpha \beta^{\prime}}{\alpha^{2} \beta^{2}}\right\}\right) \frac{d v}{\sqrt{v}}
\end{aligned}
$$

where

$$
\begin{aligned}
& \alpha^{\prime}=\frac{d}{d c} \alpha(c, v)=1-v \\
& \beta^{\prime}=\frac{d}{d c} \beta(c, v)=\frac{2-v}{2 \beta(c, v)} .
\end{aligned}
$$

The terms involving $\alpha, \beta, \alpha^{\prime}, \beta^{\prime}$ are all uniformly bounded for $v \in[0,1]$ as $c$ approaches $R$ and these bounds are independent of $g$. Combining the coefficients for the $g(\alpha)$ term, we see that the main contribution is made by the $1 / \sqrt{R-c}$ factor. Hence

$$
\frac{d}{d c} \Delta \theta(c)=2 c \int_{0}^{1} \frac{g^{\prime}(\alpha)(1-v) \sqrt{R-c}-\frac{g(\alpha)}{2 \sqrt{R-c}}(1+O(R-c))}{\alpha \beta} \frac{d v}{\sqrt{v}} .
$$

Changing back to the $r$ variable using (A1.24) gives

$$
\begin{aligned}
\frac{d}{d c} \Delta \theta(c) & =2 c \int_{c}^{R} \frac{g^{\prime}(r)\left(\frac{R-r}{R-c}\right) \sqrt{R-c}-\frac{g(r)}{2 \sqrt{R-c}}(1+O(R-c))}{r \sqrt{r+c}} \frac{d r}{\sqrt{R-c} \sqrt{r-c}} \\
& =\frac{2 c}{(R-c)} \int_{c}^{R}\left(g^{\prime}(r)(R-r)-\frac{g(r)}{2}(1+O(R-c))\right) \frac{d r}{r \sqrt{r^{2}-c^{2}}}
\end{aligned}
$$

which proves the Lemma.

\section{A.2. Attaching the cap}

To splice together the functions $r_{1}$ and $r_{2}$, we use a partition of unity function $p_{d}$ given by the following lemma. Condition (v) will insure that $K(l) \leqslant 0$ for $l \in\left(L_{1}, L_{2}\right]$.

LeMmA A2.1. There exists a $d_{0}>0$ such that for all $d>d_{0}$ there is a $C^{\infty}$ function $p_{d}(x)$ satisfying

(i) $p_{d}(x) \equiv 0, \quad x \leq 0$,

(ii) $p_{d}^{\prime}(x)>0, \quad x \in(0, d)$, 
(iii) $p_{d}^{\prime \prime}(x)>0, \quad x \in(0, d / 2) \quad$ and $\quad p_{d}^{\prime \prime}(x)<0, \quad x \in(d / 2, d)$,

(iv) $p_{d}(x) \equiv 1, \quad x \geq d$,

(v) $p_{d}(x)+p_{d}^{\prime \prime}(x) \geq 0, \quad x>0$, in particular for $x \in(d / 2, d)$.

The existence of a function $p_{1}$ satisfying (i-iv) is standard. The function $p_{d}(x)=$ $p_{1}(x / d)$, with

$$
d>d_{0}=\max _{x \in[1 / 2,1]} \sqrt{\frac{\left|p_{1}^{\prime \prime}(x)\right|}{p_{1}(1 / 2)}}
$$

will satisfy (i-v).

The arc-length coordinate $L_{2}$ should satisfy $L_{2}>d_{0}$. If necessary, scale the cap so that

$$
\begin{aligned}
L_{1} & <L_{2}-d_{0}, \\
r_{1}\left(L_{1}\right) & <r_{2}\left(L_{2}\right) .
\end{aligned}
$$

Extend $r_{1}$ in a $C^{\infty}$ function from $L_{1}$ to $L_{2}$ so that

$$
\begin{array}{rr}
r_{1}^{\prime \prime}(l)>0, & l \in\left(L_{1}, L_{2}\right] \\
\left(r_{2}-r_{1}\right)(l)>0, & l \in\left[L_{1}, L_{2}\right] \\
\left(r_{2}^{\prime}-r_{1}^{\prime}\right)(l)>0, & l \in\left[L_{1}, L_{2}\right] .
\end{array}
$$

The feasibility of (A2.3) follows from (28), of (A2.4)-(A2.5) from (30) and (A2.1)(A2.2).

Define $r(l)$ by

$$
r(l)=\left(1-p_{d}\left(l-L_{1}\right)\right) r_{1}(l)+p_{d}\left(l-L_{1}\right) r_{2}(l), \quad l \in\left[0, L_{3}\right],
$$

with $p_{d}$ as in Lemma A2.1 and $d=L_{2}-L_{1}>d_{0}$. This gives a $C^{\infty}$ metric for $l \in\left[0, L_{3}\right]$ such that $r(l)=r_{2}(l)$ for $l \in\left[L_{2}, L_{3}\right]$.

The curvature $K(l)=-r^{\prime \prime}(l) / r(l)$ will be negative in the transitional region, $l \in\left(L_{1}, L_{2}\right]$, if $r^{\prime \prime}(l)>0$. From $(\mathrm{A} 2.6)$,

$$
r^{\prime \prime}(l)=\left(1-p_{d}\right) r_{1}^{\prime \prime}+p_{d} r_{2}^{\prime \prime}+2 p_{d}^{\prime}\left(r_{2}^{\prime}-r_{1}^{\prime}\right)+p_{d}^{\prime \prime}\left(r_{2}-r_{1}\right) \text {. }
$$

All the terms in (A2.7) are positive for $l \in L_{1},\left(\left(L_{1}+L_{2}\right) / 2\right)$. For $l \in\left[\left(L_{1}+L_{2}\right) / 2, L_{2}\right]$,

$$
\begin{aligned}
r^{\prime \prime}(l) & \geq p_{d} r_{2}^{\prime \prime}+p_{d}^{\prime \prime} r_{2} & & \\
& =r_{2}\left(p_{d}+p_{d}^{\prime \prime}\right), & & \text { by }(30) \\
& >0, & & \text { by choice of } p_{d} .
\end{aligned}
$$

\section{A.3. Osserman's example}

Osserman's example has not been published. We outline the proof that on a surface with $K<0$ outside the caps and the caps given by half-spheres, the geodesic flow is ergodic.

By the symmetry of the half-sphere, a geodesic that enters the cap with angle $\phi$ will leave the cap with angle $-\phi$ having rotated exactly half-way around the cap.

Thus the flow outside the caps is the same as if we had removed the caps and abstractly identified opposite sides of the equator. Preforming this identification produces a compact Riemann surface of non-positive curvature and of rank 1. The geodesic flow on such surfaces is known to be ergodic [3]. 
From this result, we can conclude that the geodesic flow on the surface with caps is also ergodic.

\section{REFERENCES}

[1] D. V. Anosov. Geodesic flows on closed Riemann manifolds with negative curvature. Proc. Steklov Inst. Math. 90 (1960).

[2] W. Ballman, M. Brin \& K. Burns. On surfaces with no conjugate points. J. Differential Geometry 25 (1987), 249-273.

[3] W. Ballman, M. Brin \& P. Eberlein. Structure of manifolds of nonpositive curvature I. Ann. of Math. 12 (2) (1985), 171-203.

[4] V. Bangert. Mather sets for twist maps and geodesics on tori. To appear in Dynamics Reported.

[5] L. A. Bunimovich. On the ergodic properties of nowhere dispersing billiards. Commun. Math. Phys. 65 (1979), 295-312.

[6] K. Burns \& M. Gerber. Real analytic Bernoulli geodesic flows on $S^{2}$. To appear in Ergod. Th. \& Dynam. Sys. Vol. 9.

[7] M. P. DoCarmo. Differential Geometry of Curves and Surfaces, Prentice-Hall: New York, 1976.

[8] V. J. Donnay. Geodesic flow on the two-sphere with positive entropy. PhD thesis, New York University, 1986.

[9] V. J. Donnay. Geodesic flow on the two-sphere, Part II: Ergodicity. Dynamical Systems Proc., Univ. of Maryland 1986-87. J. C. Alexander (ed.). Springer Lecture Notes 1342 (1988), 112-153.

[10] P. Eberlein. When is a geodesic flow of Anosov type? J. Differential Geometry 8 (1973), 437-463.

[11] E. Hopf. Closed surfaces without conjugate points. Proc. Nat. Acad. Sci. 34 (1948), 47-51.

[12] V. F. Lazutkin. The existence of caustics for billiards in convex domains. Izvestia Acad. of Sci. Ser. Math. 37 (1) (1973), 186-216.

[13] A. Manning. Curvature bounds for the entropy of the geodesic flow on a surface. J. Loncion Math. Soc. 24 (1981), 351-357.

[14] V. I. Oseledets. A multiplicative ergodic theorem. Liapunov characteristic numbers for dynamical systems. Trans. Moscow Math. Soc. 19 (1968), 197-221.

[15] R. Osserman. Lecture at geodesic flow workshop. Cal Tech, January 1985.

[16] Ya. B. Pesin. Lyapunov characteristic exponents and smooth ergodic theory. Russ. Math. Surveys 32 (1977), 55-114.

[17] M. Wojtkowski. Invariant families of cones and Lyapunov exponents. Ergod. Th. \& Dynam. Sys. 5 (1985), 145-161.

[18] M. Wojtkowski. Principles for the design of billiards with nonvanishing Lyapunov exponent. Commun. Math. Phys. 105 (1986), 391-414. 